\title{
On modified soft rough sets on a complete atomic Boolean lattice
}

Heba I. Mustafa

\section{Correspondence:}

dr_heba_ibrahim@yahoo.com Mathematics Department, Faculty of Science, Zagazig University, Zagazig, Egypt

\begin{abstract}
Uncertainty has been used in different areas in our life such as medical diagnosis, social studies, pharmacology and others. The soft set theory and rough set theory are treated as mathematical approaches to deal with uncertainty. In 2011, Feng et al. introduced the notion of soft rough sets. In 2013, we introduced the notion of soft rough sets on a complete atomic Boolean lattice as a generalization of soft rough sets. In this paper, we strengthen the concept of soft rough set on a complete atomic Boolean lattice by defining the concept of modified soft rough set (MRS-set) on a complete atomic Boolean lattice. In this model, some properties which were not satisfied in soft rough sets on a complete atomic Boolean lattice can be proved. Finally, we will use this concept to introduce the concept of modified soft rough topology and we apply this concept in diabetes mellitus.
\end{abstract}

Keywords: Complete atomic Boolean lattice, Soft rough approximation operators on a complete atomic Boolean lattice, MSR sets on a complete atomic Boolean lattice, Boolean lattice information system, Modified soft rough topology

2010 MSC: 06B23; 54A05; 30E99

\section{Introduction}

In recent years, scientists, engineers, and mathematicians have shown great interest in uncertainty as it found many fields like decision-making, engineering, environmental science, social sciences, and medical science. Probability theory, fuzzy set theory [1], rough set theory [2,3], and other mathematical tools have been used successfully to describe uncertainty. Each of these ideas has its inherent difficulties as pointed out in $[4,5]$. Consequently, Molodtsov proposed a novel concept for modeling vagueness and uncertainty called soft set theory.

Theory of soft sets has enough parameters, so that it is free from abovementioned difficulties. It deals with uncertainty and vagueness on the one hand while on the other it has enough parametrization tools. These qualities of soft set theory make it popular among researchers and experts working in diverse areas. Some applications of soft set theory can be seen in [6-8]. Research on soft set theory is growing rapidly [9-13]. In [14], we presented soft sets on a complete atomic Boolean lattice as a generalization of soft sets and obtained the lattice structure of these soft sets.

Rough set theory, introduced by Pawlak [3] in the 1980s, is a powerful machine learning tool that has applications in many areas $[15,16]$. Rough set theory was proposed as a formal tool for modeling and processing incomplete information in information system.

(c) The Author(s). 2019 Open Access This article is distributed under the terms of the Creative Commons Attribution 4.0 International License (http://creativecommons.org/licenses/by/4.0/), which permits unrestricted use, distribution, and reproduction in any medium, provided you give appropriate credit to the original author(s) and the source, provide a link to the Creative Commons license, and indicate if changes were made. 
Pawlak rough set is mainly based on equivalence relation. But in practical, it is very difficult to find an equivalence relation among the element of a set. So, some other general relations such as tolerance one and dominance ones are considered to define rough set models. Equivalence relations can be replaced by tolerance relations [17], similarity relations [18], and binary relations [19]. The properties of the rough approximations in more general setting of complete atomic Boolean lattice were studied by Järvinen in [20]. All these proposals share the common feature that they deal with approximations of concepts in terms of granules.

The major criticism on rough set theory is that it lacks parametrization tools. In order to make parametrization tools available in rough sets, a major step is taken by Feng et al. in [21]. They introduce the concept of soft rough sets, where instead of equivalence classes parameterized subsets of a set serve the purpose of finding lower and upper approximations of a subset. In [22], Xueling and Jianming apply rough soft sets to BL-algebras. In $[23,24]$, the concept of bipolar soft rough set and bipolar soft rough relation are proposed In [25], Shabir introduced a new approach to soft rough sets called modified soft rough set (MSR-set) and studied some of their basic properties. In [14], we defined two pairs of soft rough approximation operators on a complete atomic Boolean lattice Band gave their properties. These operators suffer from unexpected properties such as soft upper approximation of non zero element might be equal zero and soft upper approximation of any element might not greater than this element. To resolve this problem, we introduce the notion of modified soft rough set on a complete atomic Boolean lattice and its application in decision making was analyzed.

This paper is arranged as follows, in "Preliminaries" section, some basic concepts of soft sets and rough sets on a complete atomic Boolean lattice are discussed. Also, we discuss the notion of soft rough set on a complete atomic Boolean lattice. The purpose of "Modified soft rough sets (MSR-sets) on a complete atomic Boolean lattice" section is to generalize soft rough set theory by introducing the notion of modified soft rough approximation operators on a complete atomic Boolean lattice B. Moreover, we study their properties and introduce a new rough set model, which is an improvement of Järvinen's model [20]. We study the relations between soft rough approximations on B [14] and current approximations. Also, we introduce the deviation of some properties of the previous soft model [14] and our new model supported by counter examples. In "Relation between MSR sets and rough sets on a complete atomic Boolean lattice" section, we introduce the notion of Boolean lattice information system. Also, the relationship between the Boolean lattice information system and soft set on a complete atomic Boolean lattice is discussed. Also, the concept of approximations of Boolean lattice information system with respect to another Boolean lattice information system is studied and we introduce an applicable example to illustrate this notion. In the last section we introduce the concept of modified soft rough topology and we apply it and its base in diabetes mellitus(DM).

\section{Preliminaries}

We assume that the reader is familiar with the usual lattice-theoretical notation and conventions, which can be found in [26, 27].

Lemma 1 [26] Let $\boldsymbol{B}=(B, \leq)$ be a complete lattice, $S, T \subseteq B$ and $\left\{X_{i}: i \in I\right\} \subseteq \wp(B)$ 
(i) If $S \subseteq T$, then $\bigvee S \subseteq \bigvee T$.

(ii) $\bigvee(S \cup T)=(\bigvee S) \bigvee(\bigvee T)$

(iii) $\bigvee\left(\bigcup\left\{X_{i}: i \in I\right\}\right)=\bigvee\left\{\bigvee X_{i} \in I\right\}$

Lemma 2 [26] Let $\boldsymbol{B}=(B, \leq)$ be a Boolean lattice, then for all $x, y \in B$

(i) $0^{\prime}=1$ and $1^{\prime}=0$,

(ii) $x^{\prime \prime}=x$,

(iii) $(x \vee y)^{\prime}=x^{\prime} \wedge y^{\prime}$, and $(x \wedge y)^{\prime}=x^{\prime} \vee y^{\prime}$,

(iv) $x \leq y$ iff $x \wedge y^{\prime}=0$.

Lemma 3 [20] Let $\boldsymbol{B}=(B, \leq)$ be a complete Boolean lattice. Then for all $\left\{x_{i}: i \in I\right\} \subseteq B$ and $y \in B$

$$
y \wedge\left(\bigvee_{i \in I} x_{i}\right)=\bigvee_{i \in I}\left(y \wedge x_{i}\right)
$$

and

$$
y \vee\left(\bigwedge_{i \in I} x_{i}\right)=\bigwedge_{i \in I}\left(y \vee x_{i}\right)
$$

Definition 1 [20] Let $\boldsymbol{B}=(B, \leq)$ be an ordered set and $x, y \in B$, we say that $x$ is covered by $y$ (or that $y$ covers $x$ ), and write, $x \prec y$ if $x<y$ and there is no element $z$ in $B$ with $x<z<y$.

Definition 2 [20] Let $\boldsymbol{B}=(B, \leq)$ be a lattice with a least element 0 . Then $a \in B$ is called an atom if $0 \prec a$. The set of atoms of $B$ is denoted by $A(B)$. The lattice $B$ is called atomic if every element of $B$ is the supremum of the atoms below it, that is $x=\bigvee\{a \in A(B): a \prec x\}$.

Definition 3 [14] Let $\boldsymbol{B}=(B, \leq)$ be a complete atomic Boolean lattice and $E$ be a set of parameters. Let $A$ be a non empty subset of $E$. A soft set over $B$, with support $A$, denoted by $f_{A}$ on $B$ is defined by the set of ordered pairs

$$
f_{A}=\left\{\left(e, f_{A}(e)\right): e \in A, f_{A}(e) \in B\right\},
$$

or is a function $f_{A}: A \rightarrow B$ s.t

$$
f_{A}(e) \neq 0 \quad \forall \quad e \in A \subseteq E \text { and } f_{A}(e)=0 \text { if } e \notin A .
$$

In other words, a soft set over $B$ is a parameterized family of elements of $B$. For each $e \in A$, $f(e)$ is considered as e-approximate element of $f_{A}$

Definition 4 [14] Let $\boldsymbol{B}=(B, \leq)$ be a complete atomic Boolean lattice. Let $A \subseteq E$ and let $f_{A}$ be a soft set over $B$. The complement of $f_{A}$, denoted by $\left(f_{A}\right)^{c}$ is defined by $\left(f_{A}\right)^{c}=\left(f^{c}, A\right)$, where $f^{c}: A \rightarrow B$ is a mapping given by $f^{c}(e)=f(e)^{\prime}$ for every $e \in A$.

Definition 5 [14] Let $\boldsymbol{B}=(B, \leq)$ be a complete atomic Boolean lattice and let $f_{A}$ be a soft set over $B$.

(i) $f_{A}$ is called full, if $\bigvee_{e \in A} f(e)=1$;

(ii) $f_{A}$ is keeping infimum, iffor any $e_{1}, e_{2} \in A$, there exists $e_{3} \in A$ such that $f\left(e_{1}\right) \wedge f\left(e_{2}\right)=$ $f\left(e_{3}\right)$; 
(iii) $f_{A}$ is keeping supremum, if for any $e_{1}, e_{2} \in A$, there exists $e_{3} \in A$ such that $f\left(e_{1}\right) \vee$ $f\left(e_{2}\right)=f\left(e_{3}\right)$;

(iv) $f_{A}$ is called partition of $B$ if

(1) $\bigvee_{e \in A} f(e)=1$,

(2) For every $e \in A, f(e) \neq 0$,

(3) For every $e_{1}, e_{2} \in A$ either $f\left(e_{1}\right)=f\left(e_{2}\right)$ or $f\left(e_{1}\right) \wedge f\left(e_{2}\right)=0$.

Definition 6 [14] Let $\boldsymbol{B}=(B, \leq)$ be a complete atomic Boolean lattice and let $f_{A}$ be a soft set over $B$. For any element $x \in B$, we define a pair of operators $x^{\vee}, x^{\wedge}: B \rightarrow B$ as follows:

$$
\begin{aligned}
& x^{\vee}=\bigvee\{b \in A(B): \exists e \in A \text { s.t } b \leq f(e) \text { and } f(e) \leq x\} \\
& x^{\wedge}=\bigvee\{b \in A(B): \exists e \in A \text { s.t } b \leq f(e) \text { and } f(e) \wedge x \neq 0\}
\end{aligned}
$$

The elements $x^{\vee}$ and $x^{\wedge}$ are called the soft lower and the soft upper approximations of $x$ over B. Two elements $x$ and $y$ are called soft equivalent if they have the same soft upper and soft lower approximations over B. The resulting equivalence classes are called soft rough sets over $B$.

Example 1 Let $B=\{0, a, b, c, d, e, f, 1\}$ and let the order $\leq$ be defined as in Fig. 1 .

The set of atoms of a complete atomic Boolean lattice $\boldsymbol{B}=(B, \leq)$ is $\{a, b, c\}$. Let $A=$ $\left\{e_{1}, e_{2}, e_{3},\right\}$ and $f_{A}$ be a soft set over $B$ defined as follows: $f\left(e_{1}\right)=a, f\left(e_{2}\right)=b$, and $f\left(e_{3}\right)=$ d. So, $f_{A}$ is not full. Many odd situation occurs. For example,

If $x=c \neq 0$, then $x^{\vee}=x^{\wedge}=0$. Also if $x=e$, then $x^{\wedge}=a \vee b=d \nsucceq e$ Moreover $c \not \leq x^{\vee}$ or $c \not \leq x^{\wedge}$ for any $x \in B$

In order to avoid these situations, we introduced in [14] the notion of full soft sets on $B$. Moreover the concept of soft postive, soft negative and soft boundary are meaningful in the case of full soft sets.

In the following, we show that negative element of any $x \in B$ cannot be avoided.

Proposition 1 Let $f_{A}$ be a soft set over $B$ which is not full. Then there exists at least $b \in A(B)$ such that $b \leq n e g(x)=\left(x^{\wedge}\right)^{\prime}$ for all $x \in B$.

Proof Since $f_{A}$ is not full, i.e., $\bigvee_{a \in A} f(a) \not \leq 1$. So $\exists b \in A(B)$ s.t $b \not \leq f(a) \forall a \in A$. Let $x \in B$ s.t $b \leq x$. If $b \leq x^{\wedge}$, then $\exists a \in A$ s.t $b \leq f(a)$ and $f(a) \wedge x \neq 0$ which is a contradiction. Hence $b \leq x^{\wedge}$. By a similar argument when $b \leq x$ it can be shown that $b \not x^{\wedge}$. Therefore $b \leq 1-x^{\wedge}=\left(x^{\wedge}\right)^{\prime}$.

\section{Modified soft rough sets (MSR-sets) on a complete atomic Boolean lattice} Some properties of generalized soft rough model

In this section, we will generalize the soft rough model on a complete atomic Boolean lattice B by defining modified soft rough sets on B. Also, we will present some properties of modified soft rough approximation operators on $\mathrm{B}$, and introduce a new soft rough model on $B$, which is an improvement of previous models $[14,20]$. 


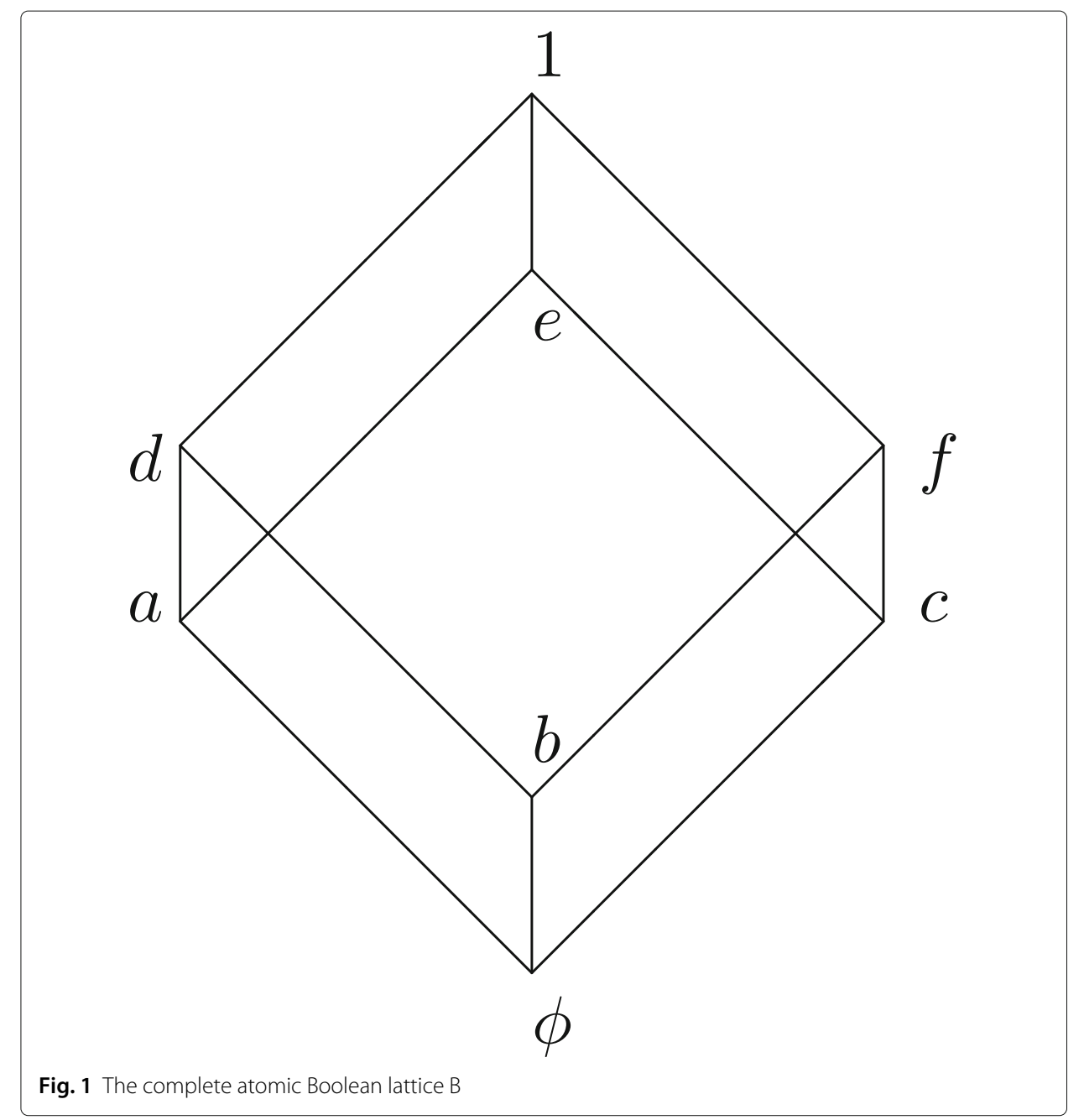

Definition 7 Let $\boldsymbol{B}=(B, \leq)$ be a complete atomic Boolean lattice and let $f_{A}$ be a soft set over B. Let $\varphi: A(B) \rightarrow \wp(A)$ be another map defined as $\varphi(b)=\{a \in A: b \leq f(a)\}$. Then the pair $(A(B), \varphi)$ is called MSR-approximation space on $B$ and for any element $x \in B$, lower MSR-approximation on $B$ is defined as

$$
\begin{aligned}
& x_{\varphi}^{\vee}=\bigvee\{a \in A(B): a \leq x, \varphi(a) \neq \varphi(b) \forall b \in A(B) \text { s.t } b \leq x\}, \\
& \text { and its upper MSR-approximation over } B \text { is defined as } \\
& x_{\varphi}^{\wedge}=\bigvee\{a \in A(B): \varphi(a)=\varphi(b) \text { for some } b \in A(b) \text { s.t } b \leq x\} . \\
& \text { If } x_{\varphi}^{\vee} \neq x_{\varphi}^{\wedge} \text {, Then } x \text { is said to be MSR-element on } B \text {. }
\end{aligned}
$$

Remark 1 Lower MSR-approximation of x over $B$ can be defined as $x_{\varphi}^{\vee}=\bigvee\{a \in A(B)$ : $\varphi(a) \neq \varphi(b) \forall b \in A(B)$ s.t $b \not x\}$ because if we let $\varphi(a) \neq \varphi(b) \forall b \in A(B)$ s.t $b \not \leq x$ and $a \not x$, then by hypothesis $\varphi(a) \neq \varphi(a)$ which is impossible.

Lemma 4 Let $\boldsymbol{B}=(B, \leq)$ be a complete atomic Boolean lattice and let $f_{A}$ be a soft set over B. Let $(A(B), \varphi)$ be a MSR-approximation space on $B$. Then for all $c \in A(B)$ and $x \in B$

(i) $c \leq x_{\varphi}^{\vee} \Longleftrightarrow c \leq x$ and $\varphi(c) \neq \varphi(b) \forall b \in A(B)$ s.t $b \not \leq x$;

ii) $c \leq x_{\varphi}^{\wedge} \Longleftrightarrow \varphi(c)=\varphi(b)$ for some $b \in A(B)$ s.t $b \leq x$. 
$\operatorname{Proof}(\mathrm{i}) \quad(\Rightarrow)$ Suppose that $c \leq x_{\varphi}^{\vee}=\bigvee\{a \in A(B): a \leq x, \varphi(a) \neq$ $\varphi(b) \forall b \in A(B)$ s.t $b \not \leq x\}$. So $c \leq x$. If $\varphi(c) \neq \varphi(b) \forall b \in A(B)$ s.t $b \not \leq x$, then $c \wedge x_{\varphi}^{\vee}=c \wedge \bigvee\{a \in A(B): a \leq x, \varphi(a) \neq \varphi(b) \quad \forall b \in A(B)$ s.t $b \not \leq x\}=$ $\bigvee\{a \wedge c: a \in A(B), c \leq x, \varphi(a) \neq \varphi(b) \forall b \in A(B)$ s.t $b \not \leq x\}$. Since $\varphi(c) \neq \varphi(b)$, then $c \neq a$, i.e., $c \wedge a=0$. Hence $c \leq\left(x_{\varphi}^{\vee}\right)^{\prime}$, a contradiction.

$(\Leftarrow)$ Suppose that $c \leq x$ and $\varphi(c) \neq \varphi(b) \forall b \in A(B)$ s.t $b \quad \leq \quad x$, then $c \leq \bigvee\{a \in A(B): a \leq x, \varphi(a) \neq \varphi(b) \forall b \not \leq x\}=x_{\varphi}^{\vee}$. Condition (ii) can be proved similarly.

Proposition 2 Let $\boldsymbol{B}=(B, \leq)$ be a complete atomic Boolean lattice and let $f_{A}$ be a soft set over B. Let $(A(B), \varphi)$ be a MSR-approximation space on B. If $\varphi(a)=\varphi(b)$ for some $a, b \in A(B)$, then for any $x \in B$ either $a, b \leq x_{\varphi}^{\wedge}$ or $a, b \not \leq x_{\varphi}^{\wedge}$.

Proof If $a \leq x_{\varphi}^{\wedge}$, then $\varphi(a)=\varphi(c)$ for some $c \in A(B)$ s.t $c \leq x$. Since $\varphi(a)=\varphi(b)$, then $\varphi(b)=\varphi(c), c \leq x$. This implies that $b \leq x_{\varphi}^{\wedge}$.

Proposition 3 Let $\boldsymbol{B}=(B, \leq)$ be a complete atomic Boolean lattice and let $f_{A}$ be a soft set over B. Let $(A(B), \varphi)$ be a MSR-approximation space on $B$. Then for all $x \in B$

(i) $x_{\varphi}^{\vee} \leq x \leq x_{\varphi}^{\wedge}$

(ii) $0_{\varphi}^{\vee}=0=0_{\varphi}^{\wedge}$

(iii) $1_{\varphi}^{\vee}=1=1_{\varphi}^{\wedge}$

Proof (i) Assume that $b \in A(B)$, s.t $b \leq x_{\varphi}^{\vee}$, then $b \leq x$. For the other inclusion, let $b \in A(B)$ s.t $b \leq x$. Then $\varphi(b)=\varphi(b)$ for some $b \leq x$. Thus $b \leq x_{\varphi}^{\wedge}$.

(ii) $0_{\varphi}^{\vee}=\bigvee\{a \in A(B): a \leq 0, \varphi(a) \neq \varphi(b) \forall b \in A(B)$ s.t $b \not \leq 0\}=0$. Also, $0_{\varphi}^{\wedge}=$ $\bigvee\{a \in A(B): \varphi(a)=\varphi(b)$ for some $b \in A(B)$ s.t $b \leq 0\}=0$. Claim (iii) can be proved similarly.

Proposition 4 Let $\boldsymbol{B}=(B, \leq)$ be a complete atomic Boolean lattice and let $f_{A}$ be a soft set over $B$. Let $(A(B), \varphi)$ be a MSR-approximation space on $B$. Then for all $x, y \in B$

(i) The mappings $\underset{\varphi}{\vee}: B \longrightarrow B$ and ${ }_{\varphi}^{\wedge}: B \longrightarrow B$ are order preserving.

(ii) The mappings $\underset{\varphi}{\vee}: B \longrightarrow B$ and ${ }_{\varphi}^{\wedge}: B \longrightarrow B$ are mutually dual.

Proof (i) Assume that $x \leq y$ and $a \leq x_{\varphi}^{\vee}$. Let $b \in A(B)$ s.t $b \not \leq y$. Since $x \leq y$, then $b \leq x$. Since $a \leq x_{\varphi}^{\vee}$, then $\varphi(a) \neq \varphi(b)$. So $a \leq y_{\varphi}^{\vee}$ and we get $x_{\varphi}^{\vee} \leq y_{\varphi}^{\vee}$. Now let $b \leq x_{\varphi}^{\wedge}$, that is $\varphi(b)=\varphi(c)$ for some $c \in A(B)$ s.t $c \leq x$. Since $x \leq y$, then $\varphi(b)=\varphi(c)$ for some $c \in A(B)$ s.t $c \leq y$. Thus $b \leq y_{\varphi}^{\wedge}$. Consequently, $x_{\varphi}^{\wedge} \leq y_{\varphi}^{\wedge}$.

(ii) We must show that $x_{\varphi}^{\wedge}=\left(\left(x^{\prime}\right)_{\varphi}^{\vee}\right)^{\prime}$ and $\left(\left(x^{\prime}\right)_{\varphi}^{\wedge}\right)^{\prime}=x_{\varphi}^{\vee}$. Let $a \in A(B)$ s.t $a \leq\left(\left(x^{\prime}\right)_{\varphi}^{\vee}\right)^{\prime}$, then $a \not \leq\left(x^{\prime}\right)_{\varphi}^{\vee}$. So, either $a \not \leq x$ or $\varphi(a)=\varphi(b)$ for some $b \in A(B)$ s.t $b \leq\left(x^{\prime}\right)^{\prime}=x$, that is $a \leq x_{\varphi}^{\wedge}$. Conversely, Let $a \leq x_{\varphi}^{\wedge}$, then $\varphi(a)=\varphi(b)$ for some $b \in A(B)$ s.t $b \leq x$. Thus $a \not \subset\left(x^{\prime}\right)_{\varphi}^{\vee}$, that is $a \leq\left(\left(x^{\prime}\right)_{\varphi}^{\vee}\right)^{\prime}$. So $x_{\varphi}^{\wedge}=\left(\left(x^{\prime}\right)_{\varphi}^{\vee}\right)^{\prime}$.

Let $a \in A(B)$ s.t $a \leq\left(\left(x^{\prime}\right)_{\varphi}^{\wedge}\right)^{\prime}$, then $\left.a \not x^{\prime}\right)_{\varphi}^{\wedge}$. Hence, $\varphi(a) \neq \varphi(b)$ for all $b \in A(B)$ s.t $b \leq x^{\prime}$. That is $a \leq x$ because otherwise, if $a \leq x^{\prime}$, then $\varphi(a) \neq \varphi(a)$, a contradiction. Thus, $a \leq x, \varphi(a) \neq \varphi(b)$ for all $b \in A(B)$ s.t $b \leq x^{\prime}$ and consequently, $a \leq x_{\varphi}^{\vee}$. 
Conversely, let $a \in A(B)$ s.t $a \leq x_{\varphi}^{\vee}$, then $a \leq x$ and $\varphi(a) \neq \varphi(b)$ for all $b \in A(B)$ s.t $b \leq x^{\prime}$. Thus $a \not \leq\left(x^{\prime}\right)_{\varphi}^{\wedge}$, i.e $a \leq\left(\left(x^{\prime}\right)_{\varphi}^{\wedge}\right)^{\prime}$. So $\left(\left(x^{\prime}\right)_{\varphi}^{\wedge}\right)^{\prime}=x_{\varphi}^{\vee}$.

For all $S \subseteq B$, we denote $S_{\varphi}^{\vee}=\left\{x_{\varphi}^{\vee}: x \in S\right\}$ and $S_{\varphi}^{\wedge}=\left\{x_{\varphi}^{\wedge}: x \in S\right\}$

Proposition 5 Let $\boldsymbol{B}=(B, \leq)$ be a complete atomic Boolean lattice and let $f_{A}$ be a soft set over $B$. Let $(A(B), \varphi)$ be a MSR-approximation space on $B$, then

i) For all $S \subseteq B, \vee S_{\varphi}^{\wedge}=(\vee S)_{\varphi}^{\wedge}$ and $\wedge S_{\varphi}^{\wedge} \geq(\wedge S)_{\varphi}^{\wedge}$.

ii) For all $S \subseteq B, \wedge S_{\varphi}^{\vee}=(\wedge S)_{\varphi}^{\vee}$ and $\vee S_{\varphi}^{\vee} \leq(\vee S)_{\varphi}^{\vee}$.

(iii) $\left(B_{\varphi}^{\wedge}, \leq\right)$ is a complete lattice; 0 is the least element and 1 is the greatest element of $\left(B_{\varphi}^{\wedge}, \leq\right)$.

(iv) $\left(B_{\varphi}^{\vee}, \leq\right)$ is a complete lattice; 0 is the least element and 1 is the greatest element $o f\left(B_{\varphi}^{\vee}, \leq\right)$.

(v) The kernal $\Theta_{\varphi}^{\vee}=\left\{(x, y): x_{\varphi}^{\vee}=y_{\varphi}^{\vee}\right\}$ of the map ${ }_{\varphi}^{\vee}: B \longrightarrow B$ is a congruence on the semi lattice $(B, \wedge)$ such that the $\Theta_{\varphi}^{\vee}$-class of any $x$ has a least element.

(vi) The kernal $\Theta_{\varphi}^{\wedge}=\left\{(x, y): x_{\varphi}^{\wedge}=y_{\varphi}^{\wedge}\right\}$ of the map ${ }_{\varphi}^{\wedge}: B \longrightarrow B$ is a congruence on the semi lattice $(B, \vee)$ such that the $\Theta_{\varphi}^{\wedge}$-class of any $x$ has a greatest element.

Proof (i) Let $S \subseteq B$. The mapping ${ }_{\varphi}^{\wedge}: B \longrightarrow B$ is order preserving, which implies that $\vee S_{\varphi}^{\wedge} \leq(\vee S)_{\varphi}^{\wedge}$ and $\wedge S_{\varphi}^{\wedge} \geq(\wedge S)_{\varphi}^{\wedge}$. Let $b \in A(B)$ and assume that $a \leq(\vee S)_{\varphi}^{\wedge}$. So, $\varphi(a)=$ $\varphi(b)$ for some $b \in A(B)$ s.t $b \leq \vee S$. So, $\varphi(a)=\varphi(b)$ for some $b \in A(B)$ and $x \in S$ s.t $b \leq x$. So $\{a \in A(B): \varphi(a)=\varphi(b)$ forsome $b \in A(B)$ s.t $b \leq \vee S\}$

$\subseteq \cup_{x \in S}\{a \in A(B): \varphi(a)=\varphi(b)$ forsome $b \in A(B)$ s.t $b \leq x\}$. Then

$(\vee S)_{\varphi}^{\wedge}=\bigvee\{a \in A(B): \varphi(a)=\varphi(b)$ for some $b \in A(b)$ s.t $b \leq \vee S\}$

$\leq \bigvee\left(\cup_{x \in S}\{a \in A(B): \varphi(a)=\varphi(b)\right.$ for some $b \in A(B)$ s.t $\left.b \leq x\}\right)$

$=\bigvee_{x \in S}(\bigvee\{a \in A(B): \varphi(a)=\varphi(b)$ for some $b \in A(b)$ s.t $b \leq x\})$ (by Lemma 1 )

$=\bigvee\left\{x_{\varphi}^{\wedge}: x \in S\right\}=\bigvee S_{\varphi}^{\wedge}$

(ii) Let $S \subseteq B$. The mapping ${ }_{\varphi}^{\vee}: B \longrightarrow B$ is order preserving, which implies that $(\wedge S)_{\varphi}^{\vee} \leq$ $\wedge S_{\varphi}^{\vee}$ and $\vee S_{\varphi}^{\vee} \leq(\vee S)_{\varphi}^{\vee}$. Let $a \in A(B)$ s.t $a \leq \wedge S_{\varphi}^{\vee}=\wedge\left\{x_{\varphi}^{\vee}: x \in S\right\}$. So, $a \leq x$ and $\varphi(a) \neq \varphi(b)$ for all $b \in A(B)$ s.t $b \leq x$ for every $x \in S$. Hence $\varphi(a) \neq \varphi(b)$ for all $b \in A(B)$ s.t $b \not \wedge \wedge$. In fact if $b \not \wedge S$, then $\exists x \in S$ s.t $b \leq x$. So, $\varphi(a) \neq \varphi(b)$. Therefore $b \leq(\wedge S)_{\varphi}^{\vee}$. Consequently, $\wedge S_{\varphi}^{\vee} \leq(\wedge S)_{\varphi}^{\vee}$. Assertions (iii) and (iv) follow easily from (i), (ii) and Proposition 3(i). The proof of (v) and (vi) follow by (i) and (ii).

The inequalities in Proposition 5 may be proper. This can be seen in the following example.

Example 2 Let $B=\{0, a, b, c, d, e, f, 1\}$ and let the order $\leq$ be defined as in Fig. 1. Let $A=\left\{e_{1}, e_{2}, e_{3},\right\}$ and $f_{A}$ be a soft set over $B$ defined as follows:

$f\left(e_{1}\right)=0, f\left(e_{2}\right)=c$, and $f\left(e_{3}\right)=d$. Then the map $\varphi$ of MSR-approximation space $(A(B), \varphi)$ on $B$ will be $\varphi(a)=\left\{e_{3}\right\}, \varphi(b)=\left\{e_{3}\right\}$, and $\varphi(c)=\left\{e_{2}\right\}$.

If we take $x=e$ and $y=f$. Then $x \vee y=1$ and $(x \vee y)_{\varphi}^{\vee}=1$. Also, $x_{\varphi}^{\vee}=c, y_{\varphi}^{\vee}=c$ and $x_{\varphi}^{\vee} \vee y_{\varphi}^{\vee}=c$. Thus $(x \vee y)_{\varphi}^{\vee}>x_{\varphi}^{\vee} \vee y_{\varphi}^{\vee}$.

Now $x_{\varphi}^{\wedge}=a \vee b \vee c=1, y_{\varphi}^{\wedge}=a \vee b \vee c=1$. So $x_{\varphi}^{\wedge} \wedge y_{\varphi}^{\wedge}=1$. On the other hand $x \wedge y=e \wedge f=c$ and $(x \wedge y)_{\varphi}^{\wedge}=0$. Thus $x_{\varphi}^{\wedge} \wedge y_{\varphi}^{\wedge}{ }_{\neq}(x \wedge y)_{\varphi}^{\wedge}$. 
Proposition 6 Let $\boldsymbol{B}=(B, \leq)$ be a complete atomic Boolean lattice and let fn be a soft set over B. Let $(A(B), \varphi)$ be a MSR-approximation space on $B$. Then, $\left(B_{\varphi}^{\vee}, \geq\right) \cong\left(B_{\varphi}^{\wedge}, \leq\right)$

Proof We show that $x_{\varphi}^{\wedge} \longrightarrow\left(x^{\prime}\right)_{\varphi}^{\vee}$ is the required dual order isomorphism. It is obvious that $x_{\varphi}^{\wedge} \longrightarrow\left(x^{\prime}\right)_{\varphi}^{\vee}$ is onto $\left(B_{\varphi}^{\vee}, \geq\right)$. We show that $x_{\varphi}^{\wedge} \longrightarrow\left(x^{\prime}\right)_{\varphi}^{\vee}$ is order embedding. Suppose that $x_{\varphi}^{\wedge} \leq y_{\varphi}^{\wedge}$. Then for all $a \in A(B), a \leq x_{\varphi}^{\wedge}$ implies $a \leq y_{\varphi}^{\wedge}$. So, for all $a \in A(B)$ such that $\varphi(a)=\varphi(c)$ for some $c \in A(B)$ s.t $c \leq x$ implies $\varphi(a)=\varphi(b)$ for some $b \in A(B)$ s.t $b \leq y$. Suppose that $\left(y^{\prime}\right)_{\varphi}^{\vee} \not\left(x^{\prime}\right)_{\varphi}^{\vee}$. So there exists $a \in A(B)$ such that $a \leq\left(y^{\prime}\right)_{\varphi}^{\vee}$ and $a \not\left(x^{\prime}\right)_{\varphi}^{\vee}$. Hence $a \leq y^{\prime}$ and $\varphi(a) \neq \varphi(b)$ for all $b \in A(B)$ s.t $b \leq\left(y^{\prime}\right)^{\prime}=y$. Also $a \not \leq\left(x^{\prime}\right)_{\varphi}^{\vee}$ implies either $a \not \leq x^{\prime}$ or $\varphi(a)=\varphi(c)$ for some $c \in A(B)$ s.t $c \leq\left(x^{\prime}\right)^{\prime}=x$, a contradiction. Hence $\left(y^{\prime}\right)_{\varphi}^{\vee} \leq\left(x^{\prime}\right)_{\varphi}^{\vee}$. On the other hand, assume that $\left(y^{\prime}\right)_{\varphi}^{\vee} \leq\left(x^{\prime}\right)_{\varphi}^{\vee}$ and $x_{\varphi}^{\wedge} \leq y_{\varphi}^{\wedge}$. So there exists $a \in A(B)$ such that $a \leq x_{\varphi}^{\wedge}$ and $a \not \leq y_{\varphi}^{\wedge}$. So $\varphi(a)=\varphi(b)$ for some $b \in A(B)$ s.t $b \leq x$. But $\varphi(a) \neq \varphi(c)$ for all $c \in A(B)$ s.t $c \leq y$. So $a \leq\left(y^{\prime}\right)_{\varphi}^{\vee}$ and $a \not \leq\left(x^{\prime}\right)_{\varphi}^{\vee}$, a contradiction.

Proposition 7 Let $\boldsymbol{B}=(B, \leq)$ be a complete atomic Boolean lattice and let $f_{A}$ be a soft set over $B$. Let $(A(B), \varphi)$ be a MSR-approximation space on $B$. Then for all $x \in B$
i) $\left(x_{\varphi}^{\vee}\right)_{\varphi}^{\vee}=x_{\varphi}^{\vee}$;
ii) $\left(x_{\varphi}^{\wedge}\right)_{\varphi}^{\wedge}=x_{\varphi}^{\wedge}$.
iii) $\left(x_{\varphi}^{\vee}\right)_{\varphi}^{\wedge}=x_{\varphi}^{\vee}$
iv) $\left(x_{\varphi}^{\wedge}\right)_{\varphi}^{\vee}=x_{\varphi}^{\wedge}$

Proof i) $\left(x_{\varphi}^{\vee}\right)_{\varphi}^{\vee}=\bigvee\left\{a \in A(B): a \leq x_{\varphi}^{\vee}, \varphi(a) \neq \varphi(b) \forall b \in A(B)\right.$ s.t $\left.b \not \leq x_{\varphi}^{\vee}\right\}$. By Proposition $3 x_{\varphi}^{\vee} \leq x$ which gives $x^{\prime} \leq\left(x_{\varphi}^{\vee}\right)^{\prime}$. So if $b \leq x^{\prime}$, then $b \leq\left(x_{\varphi}^{\vee}\right)^{\prime}$. Therefore $\left(x_{\varphi}^{\vee}\right)_{\varphi}^{\vee}=\bigvee\left\{a \in A(B): a \leq x_{\varphi}^{\vee}, \varphi(a) \neq \varphi(b) \forall b \in A(B)\right.$ s.t $\left.b \not \leq x\right\}=x_{\varphi}^{\vee}$.

ii)By Proposition $3 x_{\varphi}^{\wedge} \leq\left(x_{\varphi}^{\wedge}\right)_{\varphi}^{\wedge}$. For the reverse inclusion, let $a \leq\left(x_{\varphi}^{\wedge}\right)_{\varphi}^{\wedge}$, then $\varphi(a)=\varphi(b)$ for some $b \in A(B)$ s.t $b \leq x_{\varphi}^{\wedge}$, that is $\varphi(b)=\varphi(c)$ for some $c \in A(B)$ s.t $c \leq x$. This implies that $\varphi(a)=\varphi(c)$ for some $c \in A(B)$ s.t $c \leq x$. Hence $a \leq x_{\varphi}^{\wedge}$ and therefore $\left(x_{\varphi}^{\wedge}\right)_{\varphi}^{\wedge} \leq x_{\varphi}^{\wedge}$. Hence $\left(x_{\varphi}^{\wedge}\right)_{\varphi}^{\wedge}=x_{\varphi}^{\wedge}$.

iii) $x_{\varphi}^{\vee} \leq\left(x_{\varphi}^{\vee}\right)_{\varphi}^{\wedge}$ by Proposition 3. For the converse assume that $a \leq\left(x_{\varphi}^{\vee}\right)_{\varphi}^{\wedge}$. So $\varphi(a)=\varphi(b)$ for some $b \in A(B)$ s.t $b \leq x_{\varphi}^{\vee}$. Hence $\varphi(b) \neq \varphi(c)$ for all $c \in A(B)$ s.t $c \leq x^{\prime}$. Since $\varphi(a)=\varphi(b)$, then $\varphi(a) \neq \varphi(c)$ for all $c \in A(B)$ s.t $c \leq x^{\prime}$. Since $\varphi(a)=\varphi(a)$, then $a \leq x^{\prime}$, i.e $a \leq x$. So $a \leq x_{\varphi}^{\vee}$. Hence $\left(x_{\varphi}^{\vee}\right)_{\varphi}^{\wedge} \leq x_{\varphi}^{\vee}$ and we conclude that $\left(x_{\varphi}^{\vee}\right)_{\varphi}^{\wedge}=x_{\varphi}^{\vee}$.

iv)By Proposition $3\left(x_{\varphi}^{\wedge}\right)_{\varphi}^{\vee} \leq x_{\varphi}^{\wedge}$. Conversely, if $a \not \subset\left(x_{\varphi}^{\wedge}\right)_{\varphi}^{\vee}$, then either $a \not \leq x_{\varphi}^{\wedge}$ or $\varphi(a)=\varphi(b)$ for some $b \in A(B)$ s.t $b \leq\left(x_{\varphi}^{\wedge}\right)^{\prime}$. If $a \not \leq x_{\varphi}^{\wedge}$, then we get our required result. In later case, $\varphi(a)=\varphi(b)$ for some $b \in A(B)$ s.t $b \leq\left(x_{\varphi}^{\wedge}\right)^{\prime}$. So $\varphi(b) \neq \varphi(z)$ for all $z \in A(B)$ s.t $z \leq x$. Therefore $\varphi(a) \neq \varphi(z)$ for all $z \in A(B)$ s.t $z \leq x$. Also, $a \leq x^{\prime}$ because if $a \not x^{\prime}$, then $a \leq x$. So $\varphi(a) \neq \varphi(a)$, a contradiction. Hence $a \leq\left(x^{\prime}\right)_{\varphi}^{\vee}$. That is $a \not \leq\left(\left(x^{\prime}\right)_{\varphi}^{\vee}\right)^{\prime}=x_{\varphi}^{\wedge}$ by Proposition 4(ii).

\section{Relations between soft rough approximations on B and current approximations}

The following proposition shows a relation between soft lower approximation operators over a complete atomic Boolean lattice B and lower MSR-approximation over B.

Proposition 8 Let $\boldsymbol{B}=(B, \leq)$ be a complete atomic Boolean lattice and let $f_{A}$ be a soft set over B. Let $(A(B), \varphi)$ be a MSR-approximation space on $B$. Then for any $x \in B, x^{\vee} \leq x_{\varphi}^{\vee}$. 
Proof Let $b \in A(B)$ s.t $b \leq x^{\vee}$. Then $\exists a \in A$ s.t $b \leq f(a) \leq x$. So $a \in \varphi(b)$ and $b \leq x$. If $b \not \leq x_{\varphi}^{\vee}$, then $\varphi(b)=\varphi(c)$ for $c \in A(B)$ s.t $c \not \leq x$. Since $a \in \varphi(b)$ and $\varphi(b)=\varphi(c)$, then $a \in \varphi(c)$ which implies that $c \leq f(a) \leq x$. Hence $c \leq x$ which is a contradiction. Therefore $x^{\vee} \leq x_{\varphi}^{\vee}$.

The following example shows that the relation $\leq$ between $x_{\varphi}^{\vee}$ and $x^{\vee}$ may be proper.

Example 3 Let $B=\{0, a, b, c, d, e, f, 1\}$ and let the order $\leq$ be defined as in Fig. 1. Let $A=\left\{e_{1}, e_{2}, e_{3},\right\}$ and $f_{A}$ be a soft set over $B$ defined as follows:

$f\left(e_{1}\right)=a, f\left(e_{2}\right)=0$, and $f\left(e_{3}\right)=e$. Then the map $\varphi$ of MSR-approximation space $(A(B), \varphi)$ on $B$ will be $\varphi(a)=\left\{e_{1}, e_{3}\right\}, \varphi(b)=\phi$, and $\varphi(c)=\left\{e_{3}\right\}$.

Let $x=d$. Then $x_{\varphi}^{\vee}=a \vee b=d$ and $x^{\vee}=a$. Thus $x^{\vee}<x_{\varphi} x_{\varphi}^{\vee}$.

In the following proposition we study a necessary and sufficient condition for $x_{\varphi}^{\vee} \leq x^{\vee}$ to be hold for any $x \in B$.

Proposition 9 Let $\boldsymbol{B}=(B, \leq)$ be a complete atomic Boolean lattice and let $f_{A}$ be a soft set over $B$. Let $(A(B), \varphi)$ be a MSR-approximation space on $B$. Then for any $x \in B, x_{\varphi}^{\vee} \leq x^{\vee}$ ifffor every $b \in A(B) \exists e \in A$ s.t $f(e)=\bigvee\{a \in A(B): \varphi(a)=\varphi(b)\}$.

Proof $(\Rightarrow)$ Assume that $x_{\varphi}^{\vee} \leq x^{\vee}$ for any $x \in B$. Let $b \in A(B)$ and $x=\bigvee\{a \in A(B)$ : $\varphi(a)=\varphi(b)\}$. So $x_{\varphi}^{\vee}=\bigvee\{a \in A(B): \varphi(a)=\varphi(c)$ for some $c \in A(B) c \leq x\}=\bigvee\{a \in$ $A(B): \varphi(a)=\varphi(b)\}=x$. Since $b \leq x=x_{\varphi}^{\vee}$ and $x_{\varphi}^{\vee} \leq x^{\vee}$, then $b \leq x^{\vee}$. Therefore $\exists e \in A$ s.t $b \leq f(e)$ and $f(e) \leq x$. Also, for all $a \in A(B)$ s.t $a \leq x$, we have $\varphi(a)=\varphi(b)$, thus $e \in \varphi(b)=\varphi(a)$. So $a \leq f(e)$ and therefore $x \leq f(e)$. Consequently, $f(e)=x=\bigvee\{a \in$ $A(B): \varphi(a)=\varphi(b)\}$.

$(\Leftarrow)$ Let $x \in B$ and $b \in A(B)$ s.t $b \leq x_{\varphi}^{\vee}$. So for every $a \in A(B)$, if $\varphi(a)=\varphi(b)$, then $a \leq x$. Hence $\bigvee\{a \in A(B): \varphi(a)=\varphi(b)\} \leq x$. By assumption, $\exists e \in A$ s.t $f(e)=\bigvee\{a \in$ $A(B): \varphi(a)=\varphi(b)\}$. Hence $b \leq f(e)$ and $f(e) \leq x$. Therefore $b \leq x^{\vee}$ and consequently, $x_{\varphi}^{\vee} \leq x^{\vee}$.

Remark 2 In general, there is no relation between $x_{\varphi}^{\wedge}$ and $x^{\wedge}$. If $x=e$ in Example 1, then $x^{\wedge}=d$ and $x_{\varphi}^{\wedge}=e \not d$.

In Propositions 10 and 11 and Corollary 2 we show that there is a relation between $x_{\varphi}^{\wedge}$ and $x^{\wedge}$ if some specific conditions hold.

Proposition 10 Let $\boldsymbol{B}=(B, \leq)$ be a complete atomic Boolean lattice and let $f_{A}$ be a soft set over B. Let $(A(B), \varphi)$ be a MSR-approximation space on $B$. Then $f_{A}$ is full iff $x_{\varphi}^{\wedge} \leq x^{\wedge}$ for every $x \in B$.

Proof $(\Rightarrow)$ Assume that $f_{A}$ is full and $x \in B$. Let $a \in A(B)$ s.t $a \leq x_{\varphi}^{\wedge}$, then $\exists b \in A(B)$ s.t $b \leq x$ and $\varphi(a)=\varphi(b)$. Since $b \leq 1=\bigvee_{e \in A} f(e)$, then $\exists e \in A$ s.t $b \leq f(e)$. Hence $b \leq f(e) \wedge x$ and thus $f(e) \wedge x \neq 0$. By, $b \leq f(e)$, we have $e \in \varphi(b)=\varphi(a)$ and therefore $a \leq f(e)$. Consequently, $x_{\varphi}^{\wedge} \leq x^{\wedge}$.

$(\Leftarrow)$ Suppose that $x_{\varphi}^{\wedge} \leq x^{\wedge}$ for every $x \in B$, we show that $1 \leq \bigvee_{e \in A} f(e)$. Let $a \in A(B)$, 
then $a \leq a_{\varphi}^{\wedge} \leq a^{\wedge}$. Therefore $\exists e \in A$ s.t $f(e) \wedge a \neq 0$ and thus $a \leq f(e)$ because $a \in A(B)$. Consequently, $f_{A}$ is a full soft set over $\mathrm{B}$.

Corollary 1 Let $\boldsymbol{B}=(B, \leq)$ be a complete atomic Boolean lattice and let $f_{A}$ be a soft set over B. Let $(A(B), \varphi)$ be a MSR-approximation space on $B$. Then $f_{A}$ is full iff $x \leq x^{\wedge}$ for every $x \in B$.

Proof $(\Rightarrow)$ Assume that $f_{A}$ is full and $x \in B$. Then $x \leq x_{\varphi}^{\wedge} \leq x^{\wedge}$ (by Propositions 3 and 10).

$(\Leftarrow)$ Assume that $x \leq x^{\wedge}$ for every $x \in B$. Let $b \in A(B)$, then $b \leq b^{\wedge} \leq \bigvee\{f(e): e \in A$ and $f(e) \wedge b \neq 0\}=\bigvee\{f(e): e \in A$ and $b \leq f(e)\}$. Therefore $\exists e \in A$ s.t $b \leq f(e)$ and consequently, $f_{A}$ is full.

Proposition 11 Let $\boldsymbol{B}=(B, \leq)$ be a complete atomic Boolean lattice and let $f_{A}$ be a soft set over $B$. Let $(A(B), \varphi)$ be a MSR-approximation space on $B$. Then $x^{\wedge} \leq x_{\varphi}^{\wedge}$ for every $x \in B$ iff for every $e_{1}, e_{2} \in A, f\left(e_{1}\right) \wedge f\left(e_{2}\right)=0$ whenever $f\left(e_{1}\right) \neq f\left(e_{2}\right)$.

Proof $(\Rightarrow)$ Assume that $x^{\wedge} \leq x_{\varphi}^{\wedge}$ for every $x \in B$. Let $e_{1}, e_{2} \in A$, if $f\left(e_{1}\right) \wedge f\left(e_{2}\right) \neq 0$, then $\exists b \in A(B)$ s.t $b \leq f\left(e_{1}\right) \wedge f\left(e_{2}\right)$. Since $b \leq f\left(e_{1}\right)$, then $f\left(e_{1}\right) \leq \bigvee\{f(e): b \leq f(e)\}=$ $\bigvee\{f(e): b \wedge f(e) \neq 0\}=b^{\wedge} \leq b_{\varphi}^{\wedge}=\bigvee\{a \in A(B): \varphi(a)=\varphi(b)\}$. On the other hand we show that $\bigvee\{a \in A(B): \varphi(a)=\varphi(b)\} \leq f\left(e_{1}\right)$. Let $c \in A(B)$ s.t $c \leq \bigvee\{a \in A(B): \varphi(a)$ $=\varphi(b)\}$, then $\varphi(c)=\varphi(b)$ and thus $e_{1} \in \varphi(b)=\varphi(c)$. Therefore $c \leq f\left(e_{1}\right)$ and consequently, $f\left(e_{1}\right)=\bigvee\{a \in A(B): \varphi(a)=\varphi(b)\}$. Similarly, by $b \leq f\left(e_{2}\right), f\left(e_{2}\right)=\bigvee\{a \in$ $A(B): \varphi(a)=\varphi(b)\}$ and hence $f\left(e_{1}\right)=f\left(e_{2}\right)$.

$(\Leftarrow)$ Assume that for every $e_{1}, e_{2} \in A, f\left(e_{1}\right) \wedge f\left(e_{2}\right)=0$ whenever $f\left(e_{1}\right) \neq f\left(e_{2}\right)$. Let $x \in B$ and $a \in A(B)$ s.t $a \leq x^{\wedge}$, then $\exists e_{1} \in A$ s.t $a \leq f\left(e_{1}\right)$ and $f\left(e_{1}\right) \wedge x \neq 0$. So, $\exists$ $b \in A(B)$ s.t $b \leq f\left(e_{1}\right) \wedge x$. We show that $\varphi(a)=\left\{e_{2} \in A: f\left(e_{2}\right)=f\left(e_{1}\right)\right\}$. If $f\left(e_{2}\right) \neq f\left(e_{1}\right)$, then $f\left(e_{1}\right) \wedge f\left(e_{2}\right)=0$ by assumption. Thus $a \not \leq f\left(e_{2}\right)$ because $a \leq f\left(e_{1}\right)$ and therefore $e_{2} \notin \varphi(a)$. So $\varphi(a) \subseteq\left\{e_{2} \in A: f\left(e_{2}\right)=f\left(e_{1}\right)\right\}$. On the other hand, if $f\left(e_{2}\right)=f\left(e_{1}\right)$, then $a \leq f\left(e_{2}\right)$ and hence $e_{2} \in \varphi(a)$. Consequently, $\varphi(a)=\left\{e_{2} \in A: f\left(e_{2}\right)=f\left(e_{1}\right)\right\}$. Similarly we can show that $\varphi(b)=\left\{e_{2} \in A: f\left(e_{2}\right)=f\left(e_{1}\right)\right\}$ and thus $\varphi(a)=\varphi(b)$. Since $b \leq x$, then $a \leq x_{\varphi}^{\wedge}$.

Corollary 2 Let $\boldsymbol{B}=(B, \leq)$ be a complete atomic Boolean lattice and let $f_{A}$ be a soft set over $B$. Let $(A(B), \varphi)$ be a MSR-approximation space on $B$. If $f(e) \neq 0$ for every $e \in A$, then $f_{A}$ is a partition soft set iff $x^{\wedge}=x_{\varphi}^{\wedge}$ for every $x \in B$.

Proof Obvious.

Deviation of some properties of the previous soft rough approximations and the current approximations

In this section we will introduce the deviations of some properties of soft rough approximations on a complete atomic Boolean lattice B [14] and the current approximations.

On accounting of Proposition 3, 5, 6, and 7[parts (ii) and (iv)], there are deviations between some of the properties of lower and upper approximations on B and MSR lower and upper approximations on $\mathrm{B}$, as follows 
Let $\mathbf{B}=(B, \leq)$ be a complete atomic Boolean lattice and let $f_{A}$ be a soft set over B. Then for all $x \in B$

(i) $x \not \leq x^{\wedge}$

(ii) $1_{\varphi}^{\vee} \neq 1 \neq 1^{\wedge}$

(iii) For all $S \subseteq B, \wedge S^{\vee} \neq(\wedge S)^{\vee}$.

(iv) $\left(x^{\wedge}\right)^{\wedge} \not \leq x^{\wedge}$.

(v) $\left(x^{\vee}\right)^{\wedge} \not x^{\vee}$

(vi) In general $\left(B^{\vee}, \geq\right) \varsubsetneqq\left(B^{\wedge}, \leq\right)$

The following counter example support our claims about the above deviations.

Example 4 Let $B=\{0, a, b, c, d, e, f, 1\}$ and let the order $\leq$ be defined as in Fig. 1. Let $A=\left\{e_{1}, e_{2}, e_{3},\right\}$.

(1) Let $f_{A}$ be a soft set over $B$ defined as follows:

$f\left(e_{1}\right)=a, f\left(e_{2}\right)=b, f\left(e_{3}\right)=d$ and $f\left(e_{4}\right)=0$. So $f_{A}$ is not full. Let $x=f$, then $x^{\wedge}=d \nsucceq f$. So, $x \not x^{\wedge}$. Also, $1_{\varphi}^{\vee}=d=1^{\wedge} \neq 1$.

(2) Let $f_{A}$ be a soft set over $B$ defined as follows: $f\left(e_{1}\right)=a, f\left(e_{2}\right)=f, f\left(e_{3}\right)=e$ and $f\left(e_{4}\right)=$

0. So $f_{A}$ is not keeping infumum. Let $S=\{f, e\}$, then $\wedge S=f \wedge e=c$ and $(\wedge S)^{\vee}=c^{\vee}=0$. Also, $S^{\vee}=\{f, e\}$ and hence $\wedge S^{\vee}=f \wedge e=c \not \leq 0=(\wedge S)^{\vee}$. Let $x=a$, then $x^{\wedge}=e$ and $\left(x^{\wedge}\right)^{\wedge}=e^{\wedge}=1 \not \leq e=x^{\wedge}$. Also $x^{\vee}=a$ and $\left(x^{\vee}\right)^{\wedge}=e \not \leq a=x^{\vee}$.

Let $x=a$ and $y=f$; then $x^{\wedge}=e$ and $y^{\wedge}=1$. Therefore $x^{\wedge} \leq y^{\wedge}$. On the other hand $y^{\vee \vee}=a \nless \leq f=x^{\prime \vee}$. Hence $\left(B^{\vee}, \geq\right) \varsubsetneqq\left(B^{\wedge}, \leq\right)$.

Remark 3 (1)It is mentioned that in order to prove that $x \leq x^{\wedge}$ and $1^{\vee}=1=1^{\wedge}$ in [14] we employed a strong condition on soft set $f_{A}$ over a complete atomic Boolean lattice to be full. However in proving $x \leq x_{\varphi}^{\wedge}$ and $1_{\varphi}^{\vee}=1=1_{\varphi}^{\wedge}$ in Proposition 3 no such condition is required.

(2) Also to prove that for all $S \subseteq B, \wedge S n=(\wedge S)^{\vee}$ in [14] we employed a strong condition on soft set $f_{A}$ over a complete atomic Boolean lattice to be keeping infimum. However in proving $\wedge S_{\varphi}^{\vee}=(\wedge S)_{\varphi}^{\vee}$ in Proposition 5 no such condition is required.

(3) Finally to prove that $\left(x^{\vee}\right)^{\wedge}=x^{\vee},\left(x^{\wedge}\right)^{\wedge}=x^{\wedge}$ and $\left(B^{\vee}, \geq\right) \cong\left(B^{\wedge}, \leq\right)$ we employed a strong condition on soft set $f_{A}$ over a complete atomic Boolean lattice to be a partition. However in proving $\left(x_{\varphi}^{\vee}\right)_{\varphi}^{\wedge}=x_{\varphi}^{\vee},\left(x_{\varphi}^{\wedge}\right)_{\varphi}^{\wedge}=x_{\varphi}^{\wedge}$ and $\left(B_{\varphi}^{\vee}, \geq\right) \cong\left(B_{\varphi}^{\wedge}, \leq\right)$ in Propositions 6 and 7 no such condition is required.

(4) It is clear that MSR-element over a complete atomic Boolean lattice satisfies all the basic properties Järvinen's approximations [20]. Thus, MSR-element over a complete atomic Boolean lattice provides a good combination of roughness and parametrization.

\section{Relation between MSR sets and rough sets on a complete atomic Boolean lattice}

In the following, we introduce the notion of Boolean lattice information system and we show that every soft sets on a complete atomic Boolean lattice induces a Boolean lattice information system and vice versa.

Definition 8 Let $\boldsymbol{B}=(B, \leq)$ be a complete atomic Boolean lattice and $A$ be a finite set of attributes. The pair $(A(B), A, V, g)$ is called lattice information system, if $g$ is an 
information function from $A(B) \times A$ to $V=\bigcup_{e \in A} V_{e}$ where $V_{e}=\{g(b, e): b \in A(B), e \in$ $A\}$ is the values of the attribute set $e$.

Definition $9 \mathrm{~A}$ lattice information system $(A(B), A, V, g)$ is called Boolean lattice information system if $V=\{0,1\}$.

Definition 10 Let $\boldsymbol{B}=(B, \leq)$ be a complete atomic Boolean lattice and let $S=f_{A}$ be a soft set over $B$. Then $f_{A}$ induces a Boolean lattice information system $I_{s}=\left(A(B), A, V, g_{s}\right)$, where $g_{s}: A(B) \times A \longrightarrow V=\{0,1\}$, For any $b \in A(B)$ and $e \in A$,

$$
g_{s}(b, e)= \begin{cases}1 & \text { if } b \leq f(e), \\ 0 & \text { if } b \leq f(e)\end{cases}
$$

Definition 11 Let $\boldsymbol{B}=(B, \leq)$ be a complete atomic Boolean lattice and $I=(A(B)$, $A, V, g)$ be a Boolean lattice information system. Then $S^{I}=f_{A}^{I}$ is called a soft set over $B$ induced by I, where $f_{A}^{I}: A \longrightarrow B$ and for $e \in A, f^{I}(e)=\vee\{b \in A(B): g(b, e)=1\}$.

Proposition 12 Let $\boldsymbol{B}=(B, \leq)$ be a complete atomic Boolean lattice and $S=f_{A}$ be a soft set over $B$. Let $I_{S}=\left(A(B), A, V, g_{s}\right)$ be a Boolean lattice information system induced by $S$ and $S^{I_{S}}=f_{A}^{I_{S}}$ be a soft set over B induced by $I_{s}$. Then $f_{A}^{I_{S}}=f_{A}$.

Proof By Definition 11, for any $e \in A, f_{A}^{I_{s}}(e)=\vee\left\{b \in A(B): g_{s}(b, e)=1\right\}$.

By Definition 10, for any $b \in A(B)$ and $e \in A$,

$$
g_{s}(b, e)=\left\{\begin{array}{l}
1 \text { if } b \leq f(e), \\
0 \text { if } b \not \leq f(e)
\end{array}\right.
$$

This implies that $g_{s}(b, e)=1 \Leftrightarrow b \leq f(e)$. So, for any $b \in A(B) e \in A, f(e)=f^{I_{s}}(e)$.

Proposition 13 Let $\boldsymbol{B}=(B, \leq)$ be a complete atomic Boolean lattice and $I=$ $(A(B), A, V, g)$ be a Boolean lattice information system. Let $S^{I}=f_{A}^{I}$ be a soft set over $B$ induced by $I$ and $I_{S^{I}}=\left(A(B), A, V, g_{S^{I}}\right)$ be a Boolean lattice information system induced by $S^{I}$. Then $I=I_{S}$.

Proof By Definition 10, for any $b \in A(B)$ and $e \in A$,

$$
g_{s^{I}}(b, e)=\left\{\begin{array}{l}
1 \text { if } b \leq f^{I}(e), \\
0 \text { if } b \leq f^{I}(e)
\end{array}\right.
$$

By Definition 11, for any $e \in A, f^{I}(e)=\vee\{b \in A(B): g(b, e)=1\}$. Since $I=$ $(A(B), A, V, g)$ be a Boolean lattice information system, then $g(b, e)=0$ if $b \nless f^{I}(e)$. This implies that

$$
g(b, e)=\left\{\begin{array}{l}
1 \text { if } b \leq f^{I}(e), \\
0 \text { if } b \not f^{I}(e)
\end{array}\right.
$$

So for any $b \in A(B)$ and $e \in A, g_{s^{I}}(b, e)=g(b, e)$. Hence $g_{s^{I}}=g$ and Consequently, $I=I_{S^{I}}$. 
Definition 12 Let $\boldsymbol{B}=(B, \leq)$ be a complete atomic Boolean lattice and let $S=f_{A}$ be a soft set over $B$. Let $I_{s}=\left(A(B), A, V, g_{s}\right)$ be a Boolean lattice information system induced by $S$. Then $I_{s}$ induces a mapping $\psi_{s}: A(B) \longrightarrow B$ as follows; for every $a, b \in A(B)$

$$
a \leq \psi_{s}(b) \Leftrightarrow \forall e \in A g_{s}(a, e)=g_{s}(b, e)
$$

In [14], we define a mapping induced by a soft set $f_{A}$ on a complete atomic Boolean lattice $B$ as follows

Definition 13 [14] Let $\boldsymbol{B}=(B, \leq)$ be a complete atomic Boolean lattice and let $f_{A}$ be a soft set over $B$. Define a mapping $\psi_{f}: A(B) \rightarrow B$ by

$$
a \leq \psi_{f}(b) \Leftrightarrow \exists e \in A \text {, s.t } a \leq f(e) \text { and } b \leq f(e)
$$

for every $a, b \in A(B)$. Then $\psi_{f}$ is called the mapping induced by $f_{A}$ on $B$.

Definition 14 [14] Let $\boldsymbol{B}=(B, \leq)$ be a complete atomic Boolean lattice and let $f_{A}$ be a soft set over $B$. Let $\psi_{f}: A(B) \rightarrow B$ be the mapping induced by $f_{A}$ on $B$. We define a pair of soft approximation operators $\nabla_{f}, \Delta_{f}: B \rightarrow B$ as follows $s$

$$
\begin{aligned}
& x^{\nabla_{f}}=\bigvee\left\{b \in A(B): \psi_{f}(b) \leq x\right\}, \text { and } \\
& x^{\Delta_{f}}=\bigvee\left\{b \in A(B): \psi_{f}(b) \wedge x \neq 0\right\} .
\end{aligned}
$$

The elements $x^{\nabla_{f}}$ and $x_{f}^{\Delta_{f}}$ are called the soft lower and the soft upper approximations of $\mathrm{x}$ with respect to the mapping $\psi_{f}$ induced by $f_{A}$ respectively. Two elements $\mathrm{x}$ and $\mathrm{y}$ are called equivalent if they have the same soft upper and lower approximations with respect to the mapping $\psi_{f}$ induced by $f_{A}$ on $\mathrm{B}$. The resulting equivalence classes are called soft rough sets with respect to the mapping $\psi_{f}$ induced by $f_{A}$ on $\mathrm{B}$.

Proposition 14 Let $\boldsymbol{B}=(B, \leq)$ be a complete atomic Boolean lattice and $S=f_{A}$ be a partition soft set over $B$. Let $I_{s}=\left(A(B), A, V, g_{s}\right)$ be a Boolean lattice information system induced by $f_{A}$. Then

$$
a \leq \psi_{s}(b) \Leftrightarrow a \leq \psi_{f}(b) \forall a, b \in A(B)
$$

Proof $(\Rightarrow)$ Let $a, b \in A(B)$ s.t $a \leq \psi_{s}(b)$. Then $\forall e \in A, g_{s}(a, e)=g_{s}(b, e)$. Since $a \leq 1$ and $f_{A}$ be a partition soft set, then $\exists e \in A$ s.t $a \leq f(e)$. So $g_{s}(b, e)=g_{s}(a, e)=1$ and therefore $b \leq f(e)$. Consequently, $a \leq \psi_{f}(b)$.

$(\Leftarrow)$ Let $a, b \in A(B)$ s.t $a \leq \psi_{f}(b)$. Then $\exists e_{1} \in A$ s.t $a \leq f\left(e_{1}\right)$ and $b \leq f\left(e_{1}\right)$. So $g_{s}\left(a, e_{1}\right)=g_{s}\left(b, e_{1}\right)$. For every $e_{2} \in A-\left\{e_{1}\right\}$, if $f\left(e_{1}\right)=f\left(e_{2}\right)$, then $a \leq f\left(e_{2}\right)$ and $b \leq f\left(e_{2}\right)$ and thus $g_{s}\left(a, e_{2}\right)=1=g_{s}\left(b, e_{2}\right)$. If $f\left(e_{1}\right) \neq f\left(e_{2}\right)$, then $f\left(e_{1}\right) \wedge f\left(e_{2}\right)=0$ because $f_{A}$ is a partition. Since $a \leq f\left(e_{1}\right)$ and $b \leq f\left(e_{1}\right)$, then $a \not \leq f\left(e_{2}\right)$ and $b \not \leq f\left(e_{2}\right)$ and therefore $g_{s}\left(a, e_{2}\right)=0=g_{s}\left(b, e_{2}\right)$. So, $g_{s}(a, e)=g_{s}(b, e) \forall e \in A$ and consequently $a \leq \psi_{s}(b)$.

Proposition 15 Let $\boldsymbol{B}=(B, \leq)$ be a complete atomic Boolean lattice and let $S=f_{A}$ be a soft set over $B$. Let $(A(B), \varphi)$ be a MSR-approximation space on $B$ and $I_{s}=\left(A(B), A, V, g_{s}\right)$ be a Boolean lattice information system induced by $S$. Then

(i) $\forall a, b \in A(B) a \leq \psi_{s}(b) \Leftrightarrow \varphi(a)=\varphi(b)$. 
(ii) $\forall x \in B, x_{\varphi}^{\vee}=x_{s}^{\nabla}$, where $x_{s}^{\nabla}=\bigvee\left\{b \in A(B): \psi_{s}(b) \leq x\right\}$

(iii) $\forall x \in B, x_{\varphi}^{\wedge}=x_{s}^{\triangle}$, where $x_{s}^{\triangle}=\bigvee\left\{b \in A(B): \psi_{s}(b) \wedge x \neq 0\right\}$

Proof i) $(\Rightarrow)$ Let $a, b \in A(B)$ s.t $a \leq \psi_{s}(b)$. So $g_{s}(a, e)=g_{s}(b, e) \forall e \in A$. Let $e \in \varphi(a)$, so $a \leq f(e)$ and hence $g_{s}(a, e)=1$. Therefore $g_{s}(b, e)=1$ and thus $b \leq f(e)$. Consequently, $e \in \varphi(b)$. So $\varphi(a) \subseteq \varphi(b)$, similarly we can show that $\varphi(b) \subseteq \varphi(a)$.

$(\Leftarrow)$ Let $a, b \in A(B)$ s.t $\varphi(a)=\varphi(b)$. Let $e \in A$, if $g_{s}(a, e)=1$, then $a \leq f(e)$ and hence $e \in \varphi(a)=\varphi(b)$. Also, $b \leq f(e)$ and so $g_{s}(b, e)=1$. If $g_{s}(a, e)=0$, then $a \not \leq f(e)$ and hence $e \notin \varphi(a)=\varphi(b)$. Therefore $b \not \leq f(e)$ and thus $g_{s}(b, e)=0$. So $g_{s}(a, e)=g_{s}(b, e) \forall$ $e \in A$ and consequently, $a \leq \psi_{s}(b)$.

ii) Let $x \in B$ and $b \in A(B)$ s.t $b \leq x_{\varphi}^{\vee}$. We show that $\psi_{s}(b) \leq x$. Let $a \in A(B)$ s.t $a \leq \psi_{s}(b)$, then $\varphi(a)=\varphi(b)$ by i). But $b \leq x_{\varphi}^{\vee}$ implies that $\varphi(b) \neq \varphi(c) \forall c \in A(B)$ s.t $c \nless x$. Since $\varphi(a)=\varphi(b)$, then $a \leq x$ and therefore $\psi_{s}(b) \leq x$. This implies that $x_{\varphi}^{\vee} \leq x_{s}^{\nabla}$.

Conversely, Let $b \in A(B)$ s.t $b \leq x_{s}^{\nabla}$. So $\psi_{s}(b) \leq x$. Let $a \in A(B)$ s.t $a \leq x$, thus $a \not \leq \psi_{s}(b)$. Therefore $\varphi(a) \neq \varphi(b)$ by i) and so $b \leq x_{\varphi}^{\vee}$. Consequently, $x_{s}^{\nabla} \leq x_{\varphi}^{\vee}$.

iii) Let $x \in B$ and $b \in A(B)$ s.t $b \leq x_{\varphi}^{\wedge}$, then $\exists a \in A(B)$ s.t $a \leq x$ and $\varphi(a)=\varphi(b)$. So $a \leq \psi_{s}(b)$ by i) and therefore $\psi_{s}(b) \wedge x \neq 0$. Consequently, $b \leq x_{s}^{\Delta}$.

Conversely, let $b \in A(B)$ s.t $b \leq x_{s}^{\Delta}$, then $\psi_{s}(b) \wedge x \neq 0$. Thus $\exists a \in A(B)$ s.t $a \leq x$ and $a \leq \psi_{s}(b)$. Thus $\varphi(a)=\varphi(b)$ by i) and therefore $b \leq x_{\varphi}^{\wedge}$.

In the following, we introduce the concept of Boolean lattice information system with respect to another Boolean lattice information system. We study upper and lower MSRapproximations of soft set on a complete atomic Boolean lattice with respect to another soft set.

Definition 15 Let $\boldsymbol{B}=(B, \leq)$ be a complete atomic Boolean lattice and let $f_{A_{1}}$ be a soft set over $B$. Let $(A(B), \varphi)$ be a MSR-approximation space on $B$ where $\varphi: A(B) \longrightarrow P\left(A_{1}\right)$ is defined as $\varphi(b)=\{a \in A: b \leq f(a)\}$. Let $g_{A_{2}}$ be another soft set over $B$. For any e $\in A_{2}$, lower and upper MSR approximations of $g_{A_{2}}$ over B are denoted by $\left(g_{A_{2}}\right)_{\varphi}^{\vee}$ and $\left(g_{A_{2}}\right)_{\varphi}^{\wedge}$ defined as

$$
\begin{aligned}
& g(e)_{\varphi}^{\vee}=\bigvee\{a \in A(B): a \leq g(e), \varphi(a) \neq \varphi(b) \forall b \in A(B) \text { s.t } b \not g g(e)\} \forall e \in A_{2}, \\
& g(e)_{\varphi}^{\wedge}=\bigvee\{a \in A(B): \varphi(a)=\varphi(b) \text { for some } b \in A(b) \text { s.t } b \leq g(e)\} \forall e \in A_{2} .
\end{aligned}
$$

In order to understand this concept consider the following example:

Example 5 Let $B=\{0, a, b, c, d, e, f, 1\}$ representing 8 patients, where 0 denotes patient who drink mineral water only, a denotes patient who drink coffee, $b$ denotes patient who drink cola, $c$ denotes patient who drink tea, $d$ denotes patient who drink caffeine liquids, e denotes patient who drink antioxidant liquids, $f$ denotes patient who drink cold liquids and 1 denotes patient who drink all liquids. So, the order $\leq$ can be defined as in Fig. 1.

Let $A_{1}=\left\{e_{1}, e_{2}, e_{3},\right\}$, where $e_{1}$ denotes temperature, $e_{2}$ denotes headache and $e_{3}$ denotes stomach problem and let $f_{A_{1}}$ be a soft set over $B$ representing the diagnosis of doctor $M$, defined as follows:

$f\left(e_{1}\right)=b, f\left(e_{2}\right)=e$ and $f\left(e_{3}\right)=b$. Then the Boolean lattice information system of $f_{A_{1}}$ can be given by Table 1, where 1 and 0 denote yes and no respectively. 
Table 1 Lattice information system of the soft set $f_{A}$

\begin{tabular}{llll}
\hline & $e_{1}$ & $e_{2}$ & $e_{3}$ \\
\hline$a$ & 0 & 1 & 0 \\
$b$ & 1 & 0 & 1 \\
$c$ & 0 & 1 & 0 \\
\hline
\end{tabular}

Then the map $\varphi$ of MSR-approximation space $(A(B), \varphi)$ will be $\varphi(a)=\left\{e_{2}\right\}, \varphi(b)=$ $\left\{e_{1}, e_{3}\right\}$, and $\varphi(c)=\left\{e_{2}\right\}$.

Let $A_{2}=\left\{e_{1}, e_{2}, e_{3}, e_{4}\right\}$ and $g_{A_{2}}$ be another soft set over $B$ representing the diagnosis of doctor $N$, where $A_{2}=\left\{e_{1}, e_{2}, e_{3}, e_{4}\right\}$ and $e_{4}$ represents cough, defined as follows:

$g\left(e_{1}\right)=d, g\left(e_{2}\right)=b, g\left(e_{3}\right)=c$ and $g\left(e_{4}\right)=e$. So lower MSR-approximations of $g_{A_{2}}$ over $B$ are $g\left(e_{1}\right)_{\varphi}^{\vee}=b, g\left(e_{2}\right)_{\varphi}^{\vee}=b, g\left(e_{3}\right)_{\varphi}^{\vee}=0$ and $g\left(e_{4}\right)_{\varphi}^{\vee}=a$. Also, upper MSR-approximations of $g_{A_{2}}$ over $B$ are $g\left(e_{1}\right)_{\varphi}^{\wedge}=a \vee b \vee c=1, g\left(e_{2}\right)_{\varphi}^{\wedge}=b, g\left(e_{3}\right)_{\varphi}^{\wedge}=a \vee c=e$ and $g\left(e_{4}\right)_{\varphi}^{\wedge}=e$. So, for example the patient a will be diagnosed cough by doctor $N$.

\section{Application}

In this section, we introduce the concepts of modified soft rough topology based on the notion of modified soft lower and upper approximations

Proposition 16 Let $U$ be the universe, $X \subseteq U B=\wp(U)$ and $F_{A}$ be a soft set over $U$. Then, the collection $\tau_{S R}^{\varphi}(X)=\left\{U, \phi, X_{\varphi}^{\vee}, X_{\varphi}^{\wedge}, y y \varphi(X)=X_{\varphi}^{\wedge}-X_{\varphi}^{\vee}\right\}$, forms a topology on $U$ called the modified soft rough topology on $U$ w.r.t $X$.

Proposition 17 Let $\tau_{S R}^{\varphi}(X)$ be a modified soft rough topology on $U$ w.r.t $X$. Then the collection $\beta_{S R}^{\varphi}(X)=\left\{U, X_{\varphi}^{\vee}, B_{n} d_{\varphi}(X)\right\}$ forms a base for $\tau_{S R}^{\varphi}(X)$.

\section{Proof Obvious}

Now, we will apply the concept of soft rough topology in Diabetes mellitus (DM), commonly referred to as diabetes, is a group of metabolic diseases in which there are high blood sugar levels over a prolonged period. Symptoms of high blood sugar include frequent urination, increased thirst and increased hunger. If left untreated, diabetes can cause many complications. Acute complications can include diabetic ketoacidosis, nonketotic hyperosmolar coma or death. Serious long-term complications include heart disease, stroke, chronic kidney failure, foot ulcers, and damage to the eyes.

Consider the following information table (Table 2) giving data about 6 patients as a random representative. The rows of the table represent the attributes (the symptoms for Diabetes) and the columns represent the objects (the patients). Let $U=\left\{p_{1}, p_{2}, p_{3}, p_{4}, p_{5}, p_{6}\right\}$ and $A=\left\{e_{1}\right.$ (Frequent Urination), $e_{2}$ (Increased Hunger), $e_{3}$ (Increased Thirst) $\}$. Let $F_{A}$ be a soft over $\mathrm{U}$ given by Table 2 and $(U, \varphi)$ be a MSR-approximation space.

Let $X=\left\{p_{1}, p_{4}, p_{5}\right\}$ be the set of patients having diabetes. Then, we have $X_{\varphi}^{\vee}=\left\{p_{1}, p_{4}, p_{5}\right\}, X_{\varphi}^{\wedge}=\left\{p_{1}, p_{4}, p_{5}\right\}$ and $B n d_{\varphi}(X)=\phi$. Therefore $\tau_{S R}^{\varphi}=\left\{U, \phi,\left\{p_{1}, p_{4}, p_{5}\right\}\right\}$ is a modified soft rough topology on $\mathrm{U}$ and its soft basis $\beta_{S R}^{\varphi}(X)=\left\{U, \varphi,\left\{p_{1}, p_{4}, p_{5}\right\}\right\}$.

If the attribute frequent urination is removed, we have $X_{\varphi}^{\vee}=\left\{p_{4}, p_{5}\right\}, X_{\varphi}^{\wedge}=\left\{p_{1}, p_{3}, p_{4}, p_{5}\right\}$ and $B n d_{\varphi}(X)=\left\{p_{1}, p_{3}\right\}$. Therefore, $\tau_{S R}^{\varphi}(X)=\left\{U, \phi,\left\{p_{4}, p_{5}\right\},\left\{p_{1}, p_{3}, p_{4}, p_{5}\right\},\left\{p_{1}, p_{3}\right\}\right\}$ is a 
Table 2 Tabular representation of the soft set $F_{A}$

\begin{tabular}{lllllll}
\hline & $p_{1}$ & $p_{2}$ & $p_{3}$ & $p_{4}$ & $p_{5}$ & $p_{6}$ \\
\hline$e_{1}$ & 1 & 0 & 0 & 1 & 1 & 0 \\
$e_{2}$ & 1 & 1 & 1 & 0 & 0 & 1 \\
$e_{3}$ & 0 & 1 & 0 & 1 & 1 & 1 \\
Diabetes & 1 & 0 & 0 & 1 & 1 & 0 \\
\hline
\end{tabular}

modified soft rough topology on $\mathrm{U}$ and its soft basis $\beta_{S R}^{\varphi}(X)-e_{1}=\left\{U,\left\{p_{4}, p_{5}\right\},\left\{p_{1}, p_{3}\right\}\right\} \neq$ $\beta_{S R}^{\varphi}(X)$.

Again, if the attribute increased hunger is removed, we have $X_{\varphi}^{\vee}=\left\{p_{1}, p_{4}, p_{5}\right\}$, $X_{\varphi}^{\wedge}=\left\{p_{1}, p_{4}, p_{5}\right\}$ and $B n d_{\varphi}(X)=\phi$. Therefore, $\tau_{S R}^{\varphi}(X)=\left\{U, \phi,\left\{p_{1}, p_{4}, p_{5}\right\}\right\}$ is a modified soft rough topology on $\mathrm{U}$ and its soft basis $\beta_{S R}^{\varphi}(X)-e_{2}=\left\{U, \phi,\left\{p_{1}, p_{4}, p_{5}\right\}\right\}=\beta_{S R}^{\varphi}(X)$.

Finally, if the attribute Increased Thirst is removed, we have $X_{\varphi}^{\vee}=\left\{p_{1}, p_{4}, p_{5}\right\}$, $X_{\varphi}^{\wedge}=\left\{p_{1}, p_{4}, p_{5}\right\}$ and $B n d_{\varphi}(X)=\phi$. Therefore $\tau_{S R}^{\varphi}(X)=\left\{U, \phi,\left\{p_{1}, p_{4}, p_{5}\right\}\right\}$ is a modified soft rough topology on $U$ and its soft basis $\beta_{S R}^{\varphi}(X)-e_{3}=\left\{U, \phi,\left\{p_{1}, p_{4}, p_{5}\right\}\right\}=\beta_{S R}^{\varphi}(X)$.

Therefore, $\operatorname{Core}\left(S R^{\varphi}\right)(X)=\left\{e_{1}\right\}$, i.e., frequent urination is the key attribute that has close connection to disease diabetes.

\section{Algorithm:}

Step 1: Given a finite universe $U$, a finite set A of attributes represent the data as an information table, rows of which are labeled by attributes (C),columns by objects and entries of the table are attribute values.

Step 2: Find the lower MSR approximation, upper MSR approximation and the soft MSRboundary region of $X \subseteq U$.

Step 3: Generate the soft rough topology $\tau_{S R}^{\varphi}(X)$ on $\mathrm{U}$ and its soft basis $\beta_{S R}^{\varphi}(X)$.

Step 4: Remove an attribute $x$ from conditions of attributes $(C)$ and find the lower and upper MSR approximations and the MSR-boundary region of X on $C-(x)$.

Step 5: Generate the soft rough topology $\tau_{S R}^{\varphi}$ on $\mathrm{U}$ and its soft basis $\beta_{S R}^{\varphi}(X)-x$.

Step 6: Repeat steps 4 and 5 for all attributes in C.

Step 7: Those attributes in $\mathrm{C}$ for which $\beta_{S R}^{\varphi}(X)-x \neq \beta_{S R}^{\varphi}(X)$ forms the $\operatorname{Core}\left(S R^{\varphi}\right)(X)$.

\section{Conclusion}

Lattice is a very important structure in mathematics. In [14], we introduced the concept of soft sets on a complete atomic Boolean lattice B. We combine soft set and rough set by introducing the concept of soft rough set on B. Some shortcoming became the part of soft rough sets on B. In this paper, we introduced the concept of Modified soft rough sets(MSR) on a complete atomic Boolean lattice. Some important properties of MSR on B have been discussed. Similar results which require some strong conditions for their proof in soft rough sets on B can be proved in MSR sets without these conditions. Furthermore, we used the modified soft rough approximation operators to introduce the concept of modified soft rough topology and apply this concept in diabetes mellitus.

Acknowledgments

Not applicable

Funding

There is no sources of funding for the research. 
Availability of data and materials

It is not applicable in my paper.

\section{Authors' contributions}

The author read and approved the final manuscript.

\section{Competing interests}

The author declares that there are no competing interests.

\section{Publisher's Note}

Springer Nature remains neutral with regard to jurisdictional claims in published maps and institutional affiliations.

Received: 1 December 2018 Accepted: 12 February 2019

Published online: 18 June 2019

\section{References}

1. Zadeh, L. A.: Fuzzy sets. Inf. Control. 8, 338-353 (1965)

2. Pawlak, Z:: Rough sets. Intern. J. Comput. Inf. Sci. 11, 341-356 (1982)

3. Pawlak, Z.: Rough Sets: Theoretical aspects of reasoning about data. Kluwer Academic Publishers, Dordrecht (1991)

4. Molodtsov, D.: Soft set theory - first results. Comput. Math. Appl. 37, 19-31 (1999)

5. Molodtsov, D.: The theory of soft set. URSS Publishers, Moscow (2004). in Russian

6. Bulendr, L., Palash, D.: Bell shaped fuzzy soft sets and their application in medical diagnosis. Fuzzy Inf. Eng. 9(1), 67-91 (2017)

7. Khizar, H., Muhammad I.A., Bing, C., Xiao, Y.: A New Type-2 Soft Set: Type-2 Soft Graphs and Their Applications. Advacnes Fuzzy Syst. volune 2017 (Article ID 6162753, 17 pages)

8. Karaaslan, F.: Soft Classes and Soft Rough Classes with Applications in Decision Making. Math. Probl. Eng., 1-11 (2016). https://doi.org/10.1155/2016/1584528

9. Yang, Y., Peng, X. D.: A revised TOPSIS method based on interval fuzzy soft set models with incomplete weight information. Fundamenta Informaticae. 152, 297-321 (2017)

10. Peng, X. D., Yang, Y.: Algorithms for interval-valued fuzzy soft sets in stochastic multi-criteria decision making based on regret theory and prospect theory with combined weight. Appl. Soft Comput. 54, 415-430 (2017)

11. Cagman, N., Karaaslan, F., Enginoglu, S.: Soft Lattices. J. N. Results Sci. 1(1), 5-17 (2012)

12. Bera, S., Roy, S. K., Karaaslan, F.: Soft congruence relation over lattices. Hacet. J. Math. Stat. 46(6), 1035-1042 (2017)

13. Karaaslan, F.: Fuzzy Soft Lattice Theory. ARPN J. Sci. Technol. 3(3), 248-253 (2013)

14. Mustafa, H. I.: Soft rough approximation operators on a complete atomic Boolean lattice. Math. Probl. Eng. volume 6162013( Article ID 486321, 11 pages)

15. Huang, Y., Li, T., Luo, C., Fujita, H., Horng, S.-J.: Matrix based dynamic updating rough fuzzy approximations for data mining. Knowl.-Based Syst. 119, 273-283 (2017)

16. Hu, C., Liu, S., Huang, X.: Dynamic updating approximations in multigranulation rough sets while refining or coarsening attribute values. Knowl.-Based Syst. 130, 62-73 (2017)

17. Skowron, A., Stepaniuk, J.: Tolerance approximation spaces. Fundamenta Informaticae. 27, 245-253 (1996)

18. Slowinski, R., Vanderpooten, D.: Similarity relation as a basis for rough approximations(Wang, P., ed.), Vol. 4. Duke University Press, Duke, NC (1997)

19. Liu, G., Zhu, W.: The algebraic structures of generalized rough set theory. Inf. Sci. 178, 4105-4113 (2008)

20. Järvinen, J.: On the structure of rough approximations. Fundamenta Informaticae. 50, 135-153 (2002)

21. Feng, F., Liu, X., Leoreanu-Fotea, V., Jun, Y. B.: Soft sets and soft rough sets. Inf. Sci. 181, 1125-1137 (2011)

22. Xueling, M., Jianming, Z:: Application of rough soft sets to BL-algebras and corresponding decision making methods. J. Intell. Fuzzy Syst. 34(1), 645-658 (2018)

23. Karaaslan, F.: Bipolar soft rough relations. Commun. Fac. Sci. Univ. Ank. Ser. A1: Math. Stat. 65(1), 105-126 (2016)

24. Karaaslan, F., Gagman, N.: Bipolar soft rough sets and their applications in decision making. Africa Matematka. 29(5-6), 823-839 (2018)

25. Shabir, M., Irfan, M., Shaheen, T.: Another approach to soft rough sets. Knowl.-Based Syst. 40, 72-80 (2013)

26. Davey, B. A.: Prestley, H.A. Cambridge University Press, Cambridge (1990)

27. Gratzer, G.: General Lattice theory. Academic Press, New York (1978) 